\title{
Creating psychological tutorials on the World-Wide Web
}

\author{
JOHN H. KRANTZ and BRANDI M. EAGLEY \\ Hanover College, Hanover, Indiana
}

\begin{abstract}
The potential impact that the World-Wide Web (WWW) will have on psychology and psychological education extends far beyond serving as a resource for information acquisition. Particularly through the WWW there is the capability to develop interactive teaching resources with integrated multimedia. These resources can take the form of tutorials or quizzes. In the present paper, on-line examples illustrate how these resources can be developed and used.
\end{abstract}

The World-Wide Web (WWW) is a means of sharing documents over the Internet. As with most Internet technologies, the WWW works on the client-server model. There is one computer, called the server, where the document resides. On the user's computer is the client program, called a browser. The browser sends a request to the server for the document, and the server sends the document back to the browser. The WWW is unique in that it is a hypertext environment that allows links within and between documents, and it is capable of displaying several forms of media within the document. The formation of hypertext links and use of media is made possible by the use of a common formatting language called hypertext markup language (HTML). HTML is a simple code that is embedded in a WWW document that tells the browser how to format that document and which new document to retrieve when a hypertext link is activated (Chu, Palya, $\&$ Walter, 1995).

There are several characteristics of the WWW that make it useful for developing psychological tutorials. First, hypertext has the ability to make some part of the document sensitive to input from readers. When activated, this portion of the document becomes a link that will send readers to other locations in the same document or to other documents accessible on the Internet. These links provide readers with a tremendous control of movement within and between documents. Second, most WWW servers and browsers have image map capabilities. An image map is a figure with locations that are sensitive to the input from readers. When readers click in one of these regions, they are sent to a location linked to that region of the figure. By using image maps, readers can respond directly to graphics instead of making a translation between image and text (Krantz, 1995). Third, with the development of HTML Version 2.0, there is now the ability to use forms. A form is a means for gathering more complex input such as selections from a list or text input. Fourth, the ability to use media, such as sounds and

Correspondence should be addressed to J. H. Krantz, Psychology Department, Hanover College, P.O. Box 108, Hanover, IN 472430108 (e-mail: krantzj@hanover.edu). movies, not readily available to standard presentations, can be a big benefit to education. Many concepts in psychological education may benefit from the use of motion, and HTML allows WWW authors to link these media into the document. Finally, the ability to share our work is an important advantage of the WWW. Once created, the document can be shared with other readers around the world, and we can take advantage of what other WWW authors have developed.

Given all these features, the WWW represents a mostly unrealized resource for developing tutorials and other forms of class exercises. In that which follows, an existing set of on-line tutorials will illustrate WWW authoring techniques that can be used for educational purposes. This list of ideas is certainly not exhaustive; it merely represents some of the techniques possible and indicates ways in which they can be applied.

\section{ON-LINE TUTORIALS}

\section{Receptive Fields Tutorial}

The first tutorial to be presented is the receptive fields tutorial briefly described in Krantz (1995). The purpose of this tutorial is to help students grapple with the concept of receptive fields and some of its implications (Krantz, 1994b). The initial design of this tutorial assumed a book and/or class presentation on the topic prior to accessing the tutorial, and the tutorial served to check their comprehension of the implications of the concept. This tutorial, like all of the tutorials described in this paper, is currently and continually in revision. One likely change to this tutorial will be to add more background material to help with the comprehension of the questions.

Figure 1 shows a printout of the first part of the Receptive Fields Tutorial. It illustrates one technique that can be used to develop tutorials. Near the top of Figure 1 is a citation that shows up in this figure as being underlined. This underline is a hypertext link to the reference for that material. Frequently, finding a reference in traditional media can be difficult. The reference may be at the back of the book or at the end of chapters, and it may be awkward to find. With a hypertext link, clicking on 
The picture below illustrates how a typical experiment exploring the receptive field of a cell in the visual system might proceed. Click here to view an animation that illustrates how a receptive field might be determined (that is. mapped) in the visual system. This animation follows procedures similar to those used by Kuffler (1953) to determine the receptive fields of retinal ganglion cells. In Figure 1, notice the location of the:

1. stimulus,

2. retina in the eve, and

3. cell, in this case, in some region within the visual cortex

After studying Figure 1, either click on the area of the diagram where you think you will find the receptive field or on the name of the location in the list above.

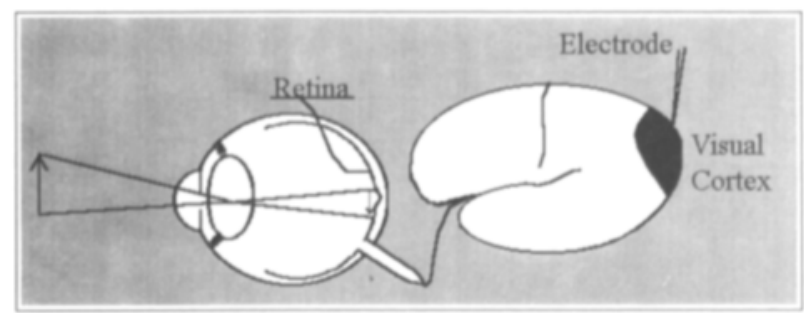

Figure 1. A portion of the Receptive Fields Tutorial (Krantz, 1994b) showing both a link to a reference and an image map. From Receptive Fields Tutorial [On-line], by J. H. Krantz, 1994. Copyright 1994 by John H. Krantz. Reprinted with permission.

the citation will take readers directly to the reference with little or no delay. Furthermore, readers can just as easily return to the original text with the use of the return arrow on the toolbar located at the top of the browser screen. Thus, examining a citation is a relatively simple activity that does not distract nearly as much from reading as it can with traditional media.

To make a citation send readers to a reference requires using HTML to set up a hypertext link. Setting up a link in HTML is nearly as easy as using one. The easiest way to begin learning how to link material in HTML is to get a program that handles the HTML. Most such programs will have authors first highlight the text that will serve as the link and then click on the link tool. Then, a box will come up and request the address or URL of the location to send readers. The basic structure of the URL is as follows:

\section{protocol://machine_name/path_to_the_document/file_name}

The protocol is the type of format for the information that the browser and server will exchange. For example, the protocol for the WWW is hypertext transfer protocol, or http, so any WWW URL will begin with http://. The machine_name is the name that uniquely identifies the server on the Internet. The name of the Hanover College psychology server is psych.hanover.edu. The path is just the name of the directories to follow to get to the document. The path to the Receptive Fields Tutorial is /Krantz/ receptive/. Finally, file_name for the beginning of the Receptive Fields Tutorial is index.html. On the Hanover College psychology server, any file with the name index.html does not have to be given. It is assumed. Thus, the full URL for the Receptive Fields Tutorial is
http://psych.hanover.edu/Krantz/receptive/; however, http://psych.hanover.edu/Krantz/receptive/index.html means the same thing.

There is one additional step to link to a particular portion of a document, which requires setting up an anchor. First, select the point within the document to which you want the reader to go. The programs will ask you for a name and ask you to enter any text you find memorable. Finally, you add \#name to the end of your URL, and this link will then take you to that location in the document. For example, in the references for the Receptive Fields Tutorial, the names used are the first author's last name. So to send readers to the reference, the end of the URL will have \#authorsname at the end of it so that readers will go directly to that reference and not to the beginning of the list.

In addition to programs that will place HTML into your document, there are some extensions to common word processors that will translate their document formats to HTML. For example, you can take a Microsoft Word for Windows document and save it directly into HTML if you have the proper extension for Word for Windows. Using these development tools, WWW authors do not even need to see the HTML.

Figure 1 also illustrates one way in which an image map can be used in a tutorial. In this particular example, the image map is used to quiz the reader about a concept related to receptive fields. The question and the figure are interrelated, and the answers are found in the figure. With an image map, readers can respond directly to the image. The figure was constructed in a paint program by using the stock elements of the program, such as circles and lines (Krantz, 1995). However, scanned images can be used as well. The figures first need to be converted to a format usable by most WWW browsers. Then the regions of the figure that will respond to user input need to be defined. The sensitive regions of an image map are in the shapes of rectangles, circles, or polygons. Several programs to help with this task are available at inexpensive shareware prices. For example, Mapedit by Boutell (1995) allows WWW developers to open the image and then identify the regions for readers' responses in the same way as the regions are drawn in any graphics program. Mapedit highlights the region being drawn to give developers feedback. Developers are then prompted for a URL to indicate where to send readers when they click on that region. The program generates the file needed by the server and authors place it in the proper location on the server. Authors can then process readers' input to an image to provide feedback.

\section{Vision and Art Tutorial}

The Vision and Art tutorial (Krantz, 1994c) is structured quite differently from the Receptive Fields Tutorial. In the Vision and Art tutorial, readers are given a great deal of text explaining some feature of the visual system, and scanned art work is then used to illustrate how art takes advantage of this feature to create specific impressions in two-dimensional art. As stated above, one of the impor- 
tant features of the hypertext environment is the ability to allow readers to move through a document in the order they desire. When it is appropriate in a tutorial to give readers this freedom, WWW authors need to develop tutorial pages using this feature of HTML. In the Vision and Art tutorial, each topic is illustrated by an icon with a diagram illustrating that feature of the visual system being discussed in that page and the title of the page. These icons are placed into a single figure, which is converted into an image map through the procedure outlined above. Now, readers can move through the tutorial in any order by simply clicking on the image map. Readers can also move through the pages in a predetermined order. At the bottom of each page are arrows that allow readers to move page by page through the tutorial both forward and backward. In between the two arrows on each page is an icon that takes readers to an index page with the image map to allow a random access of the pages.

This tutorial also illustrates the use of linking to media not available in traditional media. Embedded in the first topic, labeled "Depth Cues," is a short video. The purpose of the page is to define depth cues and describe some of the different classes of depth cues such as monocular versus binocular (Coren, Ward, \& Enns, 1994). In addition, this page illustrates some of the depth cues that are not available to the painter who tries to represent depth on the page. One of these depth cues is monocular parallax, which is the apparent speed with which objects move in relation to us as we move. To illustrate this concept, this page links to a simulated flight through a canyon, which is in what is known as an MPEG format, a format for storing and sharing video over the Internet. It would be very difficult to illustrate the concept of motion parallax without this link. In this case, the link is to an existing movie on the Internet. Thus, WWW authors can take advantage of formats other than text and still images to help readers understand the concepts being discussed.

\section{Fourier Analysis Tutorial}

The Pictorial Introduction to Fourier analysis (Krantz, 1994a) was designed to assist students in coming to grips with the conceptual basis of Fourier analysis. Fourier analysis is a mathematical procedure for taking complex wave forms, such as any sound, and determining what set of basic components can be added together to make that complex wave form. In most applications of Fourier analysis, the basic components that are thought to compose complex wave forms are sine waves. For example, in audition, simple tones are described as sine waves; most tones that reach our ear are composed of several of these tones. Yet the ear can separate these complex tones and hear the different pitches associated with the simple tones that make up the complex. Fourier analysis is useful as an analogy for understanding this ability (Plomp, 1964). Also, in the visual system, luminance changes across an object when plotted as luminance against position can be thought of as a complex wave form. Certain aspects of visual functioning can be understood by suggestions that the visual system breaks this complex wave form down into simpler components, just as in Fourier analysis (Blakemore \& Campbell, 1969). One example of the usefulness of this analogy has contributed to the understanding of the Blakemore-Sutton effect (Blakemore \& Sutton, 1969).

This tutorial illustrates another use of image maps. Figure 2 shows the result of a Fourier analysis in what is termed a frequency domain diagram. In such a diagram, the ordinate is the frequency of the sine waves and the abscissa is the amplitude of that sine wave. The $x$ position of the bar indicates the number of cycles of the sine waves per unit of space, and the $y$ height of the bar indicates the height of the sine wave. This figure is actually an image map that allows readers to examine these results at their own pace. Clicking on any bar will send readers a page with a figure that shows the fundamental sine wave - the sine wave with the lowest frequency and largest amplitude - and the sine wave indicated by the bar on which they have clicked. The reason for presenting both the fundamental and the particular sine wave is to allow readers to compare the two components of the complex wave form. Clicking on the plus sign above a particular bar gives the addition of all the sine waves from the lowest frequency up to that point, showing how the addition of more and more components gradually produces the complex wave form. Readers can examine any or all aspects of this figure until they feel comfortable with the conceptual basis of Fourier analysis. Readers are not led through the material in a prespecified set of steps that works for the author or at a predetermined pace but are required to personally decide on the order and pace that suits them best. A quiz using both hypertext links and image maps follows this section of the tutorial, which allows readers to test their own understanding of Fourier analysis. The responses to their selections provide readers with feedback to allow them to check their understanding.

\section{Basic Neural Processes Tutorials}

The tutorials on the nervous system illustrated some different features of HTML that can be used for the construction of on-line tutorials: forms and animations. Forms

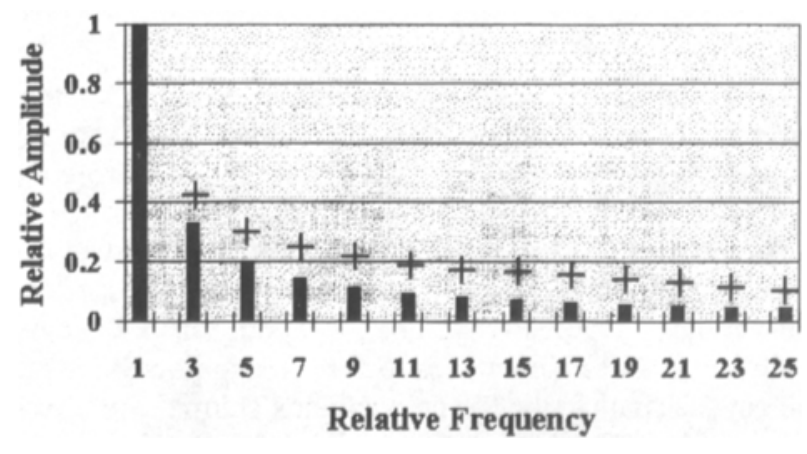

Figure 2. A portion of the Fourier analysis tutorial (Krantz, 1994a) showing an image map of a frequency domain diagram. From A Pictorial Introduction to Fourier Analysis [On-line], by J. H. Krantz, 1994. Copyright 1994 by John H. Krantz. Reprinted with permission. 
are WWW pages that allow readers to submit more complex information than simply the selection from a predetermined set of options, which is the nature of communication back to a server in hypertext links and image maps. There are several types of input in a form. The basic text input line is useful for gathering an e-mail address or simple one-word answers. Check boxes allow readers to select any number of the options, while radio buttons allow only one of the options to be selected. Check boxes are useful in a quiz if more than one option is correct, but radio buttons are preferred if readers must choose only one of the options. There is also a larger text box that allows for more extensive text input than that allowed by the simple text line. Most editors for HTML will allow the user to create these form elements along with all the other aspects of a Web page discussed above.

Given the complexity of the input and the fact that multiple input can be submitted at the same time, forms can easily be adapted to quizzes. In the Basic Neural Processes tutorials, there are pages that allow readers to test their knowledge of both neuron and brain structures. In the quiz on the structure of a neuron, a picture of a neuron is displayed, with various portions of the neuron highlighted by a number. On the Web page, the numbers are again displayed with input lines where readers can enter their guesses as to the name of the indicated structures. Check boxes or radio buttons could be used to turn this quiz into a multiple choice format if desired. There is a submit button for readers to click on to enter their responses when they have completed the quiz.

One difficulty with using forms is that the server software does not have the capability built in to process the input from a form. The server calls an additional program or set of commands, called a script, that is specified by the form. This script is then passed the input from the form by the standard method called the commongateway interface (CGI). Only the aspects of CGI necessary for understanding how forms might be used to develop scripts will be discussed here. The elements of the CGI are discussed more thoroughly by Kieley (1996). Many standardized routines have been developed to minimize the programming effort of WWW authors. One such program is Polyform by Mark Bracewell (1995), which runs on a Windows-based WWW server. This program uses a set of standard actions to process the scripts. A script in this context is the set of actions you want enacted upon your form input. For example, Polyform can e-mail the information from the form anywhere or return it in a standard Web page or store it on a disk file while returning an acknowledgment of the action. The user of Polyform determines the set of actions via a standard easy-to-use Windows interface. The interface is capable of even calling up an HTML editor so that you can more easily create the form or the standard format for returning responses to readers. Thus, the load of using forms is on the development of the form and the determination of what to do with the input and not on creating the program to handle the input. In the quizzes on the structure of a neuron, Polyform returns the correct answers with the answers entered to readers so that they can check their own results. I do not have the answers submitted to me, but a single check mark on the Polyform interface would change that.

The other added feature of the Basic Neural Processes tutorials is the use of author-developed animations. There are now CGI programs that take advantage of one of the unique features of the WWW browser Netscape Versions $1.1 \mathrm{~N}$ and later. The feature is a mime type unique to this browser. A mime type is a piece of information indicating what format the document is in. For example, all Web documents written in HTML (Chu et al., 1995) will have an extension on the file name of .htm or .html so that the browser can know how to interpret the file. There is a specific mime type for the video mentioned above. All files of MPEG video should have the extension .mpg. Netscape has developed a mime type that allows the server to display a sequence of images on the browser. If you draw the images with small changes, readers will perceive the images as moving and you have created an animation. Authors create the images and name them in a fashion determined by the animation CGI program so that it knows the order of the images and so that whenever the page is accessed with the animation, it can be presented to readers.

One of the Basic Neural Processes tutorials is an examination of some of the physical factors behind the action potential. One such factor is the attraction of oppositely charged particles. When the sodium channels on the axon membrane open during the depolarization phase, the inside of the axon is relatively more negative than the outside of the membrane, which draws the positively charged sodium ions. This particular animation simply displays a sequence of images where a positive and negative particle move together. Each image in the sequence was created by cutting and pasting the drawings of the two particles so that they were closer together in successive images until they came into contact.

\section{CONCLUSIONS}

The WWW allows the development of reader-oriented instructional exercises. The use of hypertext and image maps enables readers to move through these tutorials at their choosing. Forms allow readers to give more sophisticated responses and receive more detailed responses. Animations allow authors to take advantage of motion to capture interest and illustrate concepts that can only be illustrated by motion.

In addition, there are numerous programs to facilitate the development of these tutorials. There are programs that help in the development of HTML and process forms and take advantage of animations. Most of these programs consist of inexpensive shareware.

One of the most compelling reasons to develop these tutorials is the ease of sharing what we have developed and of using what others have developed. During the month of October 1995, all but the Pictorial Introduction to Fourier Analysis received more than 300 visits, and 
only a small proportion of that can be attributed to students at Hanover College. The Fourier tutorial received nearly 200 hits on its own. In addition to the tutorials discussed in this paper, there are tutorials in auditory perception (Welch \& Krantz, 1996) and visual illusions (Landrigan, 1995). The American Psychological Society keeps a page of tutorials of which it has knowledge at http://psych.hanover.edu/Krantz/tutor.html. With this ability to share Web tutorials, instructors will not have to rely as exclusively on their own efforts to develop exercises in fields outside their own areas of special expertise but can take advantage of the efforts of experts in these other areas.

\section{REFERENCES}

Blakemore, C., \& CAMPBELl, F. W. (1969). On the existence of neurones in the human visual system selectively sensitive to the orientation and size of retinal images. Journal of Physiology, 203, 237-260.

Blakemore, C., \& Sutton, P. (1969, October 10). Size adaptation: A new aftereffect. Science, 166, 245-247.

BoutelL, T. (1995). Mapedit [On-line]. Available URL: http://sunsite. unc.edu/boutell/mapedit/mapedit.html

Bracewell, M. C. (1995). Polyform files [On-line]. Available URL: http://wgg.com/files/PolyForm/
ChU, J. Y. M., PALYA, W. L., \& Walter, D. E. (1995). Creating a hypertext markup language document for an information server. $B e$ havior Research Methods, Instruments, \& Computers, 27, 200-205.

Coren, S., WARD, L. M., \& ENNS, J. T. (1994). Sensation and perception (4th ed.). New York: Harcourt Brace.

KIELEY, J. (1996). CGI scripts: Gateways to World-Wide Web power. $B e$ havior Research Methods, Instruments, \& Computers, 28, 165-169.

KRANTZ, J. H. (1994a). A pictorial introduction to Fourier analysis [On-line]. Available URL: http://psych.hanover.edu/Krantz/fourier' Krantz, J. H. (1994b). Receptive fields tutorial [On-line]. Available URL: http://psych.hanover,edu/Krantz/receptive/

KraNTZ, J. H. (1994c). Vision and art [On-line]. Available URL: http:// psych.hanover.edu/Krantz/art/

KranTZ, J. H. (1995). Linked Gopher and World-Wide Web services for the American Psychological Society and Hanover College psychology department. Behavior Research Methods, Instruments, \& Computers, 27, 193-197.

LANDRIGAN, D. (1995). Illusion gallery [On-line]. Available URL: http://aspen.uml.edu/ landrigad/illusion.html

Plomp, R. (1964). The ear as a frequency analyzer. Journal of the Acoustical Society of America, 36, 1628-1637.

Welch, N., \& KrantZ, J. H. (1996). The World-Wide Web as a medium for psychoacoustical demonstration and experiments: Experience and results. Behavior Research Methods, Instruments, \& Computers, 28, 192-196.

(Manuscript received November 27, 1995; revision accepted for publication January 30, 1996.) 